\title{
EMBEDDED MINIMAL SURFACES IN 3-MANIFOLDS WITH POSITIVE SCALAR CURVATURE
}

\author{
J. H. RUBINSTEIN
}

\begin{abstract}
Let $M$ be a closed orientable Riemannian 3-manifold with positive scalar curvature. We prove that any embedded closed minimal surface in $M$ has a topological description as a generalized Heegaard surface. Also an existence theorem is proved which gives examples of such minimal surfaces.
\end{abstract}

0. Let $M^{3}$ be a closed orientable 3-manifold equipped with a Riemannian metric with positive scalar curvature. A closed surface $L \subset M$ is called minimal if the mean curvature of $L$ is zero everywhere. This is equivalent to the condition that, for all variations $L_{t}$ of $L$, the area $A_{t}$ of $L_{t}$ is stationary at $L$, i.e., $A_{0}^{\prime}=0$.

Our aim is to give a simple topological description of such surfaces $L$ and to establish an existence result yielding some examples to illustrate the various cases that can arise. We now give a brief survey of previous work in this area.

We call $L \subset M$ a Heegaard surface if $M-L$ has 2 components whose closures are handlebodies. Heegaard surfaces $L, L^{\prime} \subset M$ are said to be equivalent if there is a diffeomorphism $\phi: M \rightarrow M$ with $\phi(L)=L^{\prime}$. Lawson [11] showed that if $M$ is a Riemannian $S^{3}$ with positive Ricci curvature then any embedded minimal surface is a Heegaard surface. Note also that Waldhausen [19] proved that any two Heegaard surfaces of the same genus in $S^{3}$ are equivalent. Lawson [10] gave examples of embedded minimal surfaces of every genus in $S^{3}$ with the standard metric. In addition, many such examples are constructed in [10] for other 3-manifolds with constant curvature 1 , i.e. of the form $S^{3} / \Gamma$ where $\Gamma \subset \operatorname{SO}(4)$ acts freely on $S^{3}$. Amongst these are pairs of embedded minimal surfaces in $S^{3}$ with the same genus for which there is no isometry of $S^{3}$ taking one onto the other.

More recently, Meeks-Simon-Yau (see $\S 8$ of [12]) established the same result as Lawson [11] for any $M^{3}$ with positive Ricci curvature. Also they obtained a topological characterization of orientable closed minimal surfaces embedded in $\#_{i=1}^{n} S^{2} \times S^{1}$ equipped with a Riemannian metric with nonnegative scalar curvature (see $\$ 10$ of [12]). We will give similar results for any $M$ with a metric with positive scalar curvature (or nonnegative scalar curvature if $M$ does not admit a flat metric) and will also treat nonorientable minimal surfaces.

Received by the editors June $19,1984$.

This paper was presented at the Summer Research Institute of the Australian Math. Soc. in January 1981 at Hobart, Tasmania.

1980 Mathematics Subject Classification. Primary 53A10.

Key words and phrases. Minimal surface, generalized Heegaard surface, positive scalar curvature. 
1. In this section, we collect together some related results and definitions.

THEOREM $1[6,18]$. Let $M$ be a closed orientable 3-manifold which admits a Riemannian metric with positive scalar curvature. Then $M$ can be written as a connected sum $M_{1} \# \cdots \# M_{k}$, where for each $i$ either $M_{i}$ is a copy of $S^{2} \times S^{1}$ or $\pi_{1}\left(M_{i}\right)$ is finite.

REMARK. It is conjectured that every closed 3-manifold with finite fundamental group is diffeomorphic to a standard spherical 3-manifold with constant curvature 1. This has been proved by Hamilton [8] if the 3-manifold has a metric with positive Ricci curvature.

TheOREM $2[5,16]$. Any 3-manifold $M$ which is a finite connected sum of copies of $S^{2} \times S^{1}$ and of standard spherical 3-manifolds admits a Riemannian metric with positive scalar curvature.

We now state a result which is proved in [17] for closed 3-manifolds. The same argument clearly works in the case of compact 3-manifolds with suitable boundary.

TheOREM 3 [17]. Let $M$ be a compact 3-manifold with a Riemannian metric with positive scalar curvature and assume that $\partial M$ has nonnegative mean curvature with respect to the outward normal. Then $\pi_{1}(M)$ has no subgroups of the form $\pi_{1}(J)$, where $J$ is a closed orientable surface with genus $>0$.

DEFinition (CF. [14]). A closed nonorientable surface $K$ embedded in a closed orientable 3-manifold $M$ is called a one-sided Heegaard surface if $M-K$ is an open handlebody.

REMARK. If $K \subset M$ is a one-sided Heegaard surface then $M$ has a double covering $\tilde{M}$ so that the preimage of $K$ in $\tilde{M}$ is a Heegaard surface $\tilde{K} \subset \tilde{M}$ (cf. [14] and §2).

Definition. (1) Suppose $L^{2} \subset M^{3}$ where $L, M$ are closed and orientable. $L$ is called a partial Heegaard surface for $M$ if one of the following holds:

(a) $L$ separates $M$ into 2 regions, each of which is a connected sum of an open handlebody with some closed 3-manifold.

(b) $M-L$ is the connected sum of 2 open handlebodies with a closed 3-manifold.

(2) If $K$ is a closed nonorientable surface then $K \subset M$ is a one-sided partial Heegaard surface in $M$ if $M-K$ is the connected sum of an open handlebody with a closed 3-manifold.

Remarks. By Kneser [9] and Milnor [13], any closed orientable $M^{3}$ can be uniquely expressed as a finite connected sum of prime 3-manifolds. (A closed orientable 3-manifold $Q$ is prime if either $Q=S^{2} \times S^{1}$ or any embedded $S^{2}$ in $Q$ bounds a 3-cell.) Hence, if $L$ is a separating partial Heegaard surface, then $M$ has a prime factorization $M=M_{1} \# \cdots \# M_{k+r+s}$ where $L$ can be viewed as a Heegaard surface for $M_{1} \# \cdots \# M_{k}, M_{k+1} \# \cdots \# M_{k+r}$ lies on one side of $L$ and $M_{k+r+1} \# \cdots \# M_{k+r+s}$ is on the other side. Similarly, if $L$ is nonseparating, $M$ has a prime decomposition $M=M_{1} \# \cdots \# M_{k+r} \# S^{2} \times S^{1}$ where $L$ is a Heegaard surface for $M_{1} \# \cdots \# M_{k}$ and the $S^{2} \times S^{1}$ factor arises by forming a connected sum of the two open handlebodies which are the components of $M_{1} \# \cdots \# M_{k}-$ 
$L$. The other factors $M_{k+1}, \ldots, M_{k+r}$ all miss $L$. Finally if $K$ is a one-sided partial Heegaard surface in $M$, then $M=M_{1} \# \cdots \# M_{k+r}$ where each $M_{i}$ is prime, $K$ is a one-sided Heegaard surface for $M_{1} \# \ldots \# M_{k}$ and the other factors $M_{k+i}$, for $1 \leqslant i \leqslant r$, are all disjoint from $K$.

DEFINITION. A closed surface $L \subset M$ of genus $>0$ is called incompressible if there is no disk $D \subset M$ with $D \cap L=\partial D$ a noncontractible loop on $L$. A 2-sphere $S \subset M$ is incompressible if $S$ does not bound a 3-cell in $M$.

2. Suppose $K$ is a closed nonorientable surface embedded in a closed orientable 3-manifold $M$. We will construct various double coverings $\tilde{M}$ of $M$ for which the preimage $\tilde{K}$ of $K$ in $\tilde{M}$ is orientable. Let $M=N(K) \cup Y$, where $N(K)$ is a small closed regular neighbourhood of $K$ and $Y=M-$ int $N(K)$. Let $\tilde{K}$ be the orientable double covering of $K$ and let $\tilde{Y}$ be any double covering of $Y$ such that $\partial \tilde{Y}$ is disconnected. We allow the possibility that $\tilde{Y}$ itself is disconnected and, in this case, $\tilde{Y}$ is the disjoint union of two copies of $Y$. Note that a connected double covering $\tilde{Y}$ of $Y$ exists if and only if the map $H_{1}\left(K, Z_{2}\right) \rightarrow H_{1}\left(M, Z_{2}\right)$ induced by inclusion is not onto. There is a double covering of $N(K)$ by $\tilde{K} \times[-1,1]$ with the preimage of $K$ equal to $\tilde{K} \times\{0\}$, since $N(K)$ is a twisted line bundle over $K$.

The double covering $\tilde{M}$ is obtained by gluing $\tilde{K} \times[-1,1]$ to $\tilde{Y}$ by a diffeomorphism $\psi: \tilde{K} \times\{-1,1\} \rightarrow \partial \tilde{Y}$. $\psi$ is chosen so that the double covering projections $\tilde{K} \times[-1,1] \rightarrow N(K)$ and $\tilde{Y} \rightarrow Y$ match up. So we obtain $\tilde{M}=\tilde{K} \times[-1,1] \cup_{\psi} \tilde{Y}$ and there is a double covering $p: \tilde{M} \rightarrow M$ with $p^{-1}(K)=\tilde{K} \times\{0\}$.

3. Theorem 4. Suppose $M$ is a closed orientable Riemannian 3-manifold. Assume that the scalar curvature is positive, or if $M$ admits no flat metric then it suffices to suppose that the scalar curvature is nonnegative. If $L \subset M$ is minimal then $L$ is a partial Heegaard surface for $M$.

Proof. This will follow by applying Theorem 3 to $M$ split along $L$. Firstly, if $L$ is orientable and nonseparating, we obtain a connected compact manifold $M_{1}$ with two copies of $L$ in $\partial M_{1}$, by dividing $M$ along $L$. If $L$ separates $M$, two compact manifolds $M_{2}$ and $M_{3}$ are constructed with $\partial M_{2}$ and $\partial M_{3}$ both a copy of $L$, by splitting $M$ along $L$. Finally, if $L$ is nonorientable, let $p: \tilde{M} \rightarrow M$ be the double covering constructed in $\S 2$, where $\tilde{M}=\tilde{L} \times[-1,1] \cup_{\psi} \tilde{Y}$ and $\tilde{Y}$ is disconnected. Let $Y_{1}, Y_{2}$ be the components of $\tilde{Y}$ with $\partial Y_{1}=\psi(\tilde{L} \times\{-1\})$. The compact manifold $M_{4} \subset \tilde{M}$ given by $M_{4}=\tilde{L} \times[-1,0] \cup Y_{1}$ satisfies $\partial M_{4}$, is a copy of $\tilde{L}$ and $p$ maps int $M_{4}$ diffeomorphically onto $M-L$.

By Theorem 3, there are no subgroups of $\pi_{1}\left(M_{i}\right)$ of the form $\pi_{1}(J)$, where $J$ is a closed orientable surface of positive genus and $1 \leqslant i \leqslant 4$. Note that if $M$ is assumed only to have a metric of nonnegative scalar curvature, then as in [17] the metric can be approximated by one with positive scalar curvature (since $M$ admits no flat metric) and Theorem 3 applies.

To complete the proof we apply Dehn's lemma and the Loop theorem to the surfaces $\partial M_{i}$, for $1 \leqslant i \leqslant 4$. Suppose $G$ is a component of $\partial M_{i}$ and $\left\{D_{u}: 1 \leqslant u \leqslant v\right\}$ is a maximal family of disjoint compressing disks for $G$, i.e. $\partial D_{u} \subset G$, int $D_{u} \subset$ int $M_{i}$, 
$\partial D_{u}$ is noncontractible in $G$ for all $u$ and no two curves $\partial D_{u}$ and $\partial D_{u}$. are parallel on $G$, for $u \neq w$. Let $N=N\left(\cup_{u} D_{u} \cup G\right)$ be a small closed regular neighbourhood in $M_{i}$. If some component $J$ of $\partial N-G$ has genus $>0$, then since $\pi_{1}(J) \subset \pi_{1}\left(M_{i}\right)$ (by Dehn's lemma and the Loop theorem) we get a contradiction to Theorem 3. So $\partial N$ consists of $G$ together with 2-spheres. It follows immediately that $M_{2}, M_{3}$ and $M_{4}$ are all connected sums of a handlebody ( $N$ with cells attached along the 2-spheres in $\partial N$ ) with a closed 3-manifold. Similarly, $M_{1}$ is a connected sum of two handlebodies with a closed 3-manifold, and so $L$ is a partial Heegaard surface in all cases.

REMARKS. (1) In the case that $M^{3}=\#_{i=1}^{n} S^{2} \times S^{1}$, Theorem 4 gives the results in $\$ 10$ of [12]. By results of Haken [7] and Waldhausen [19], any two orientable partial Heegaard surfaces of the same genus in $\#_{i=1}^{n} S^{2} \times S^{1}$ are equivalent if they are both separating or both nonseparating and if there are the same numbers of $S^{2} \times S^{1}$ factors in the components of the complements of the surfaces (cf. [12, §10] also).

(2) Engmann [4] and Birman [1] have given examples of two Heegaard surfaces of genus 2 in a connected sum of lens spaces $L(p, q) \# L\left(p^{\prime}, q^{\prime}\right)$ which are not equivalent. On the other hand, Bonahon and Otal [3] have recently shown that any two Heegaard surfaces with the same genus in any lens space $L(p, q)$ are equivalent.

4. Theorem 5. Suppose $M$ is a closed orientable Riemannian 3-manifold with no orientable incompressible surfaces of genus $>0$. If $H_{1}(M, Z)$ has 2-torsion then $M$ has an embedded minimal nonorientable surface which is a one-sided partial Heegaard surface. Consequently, there are double covers $\tilde{M}$ of $M$ with orientable minimal partial Heegaard surfaces.

Proof. Since $H_{1}(M, Z)$ has 2-torsion, we can choose a torsion element $\alpha \in$ $H_{1}(M, Z)$ such that $\alpha \otimes 1 \neq 0$ in $H_{1}(M, Z) \otimes Z_{2}$. Exactly as in [14], a nonorientable incompressible surface $K \subset M$ can be found so that the intersection number $\bmod 2$ of the class of $K$ in $H_{2}\left(M, Z_{2}\right)$ and $\alpha$ is one. Moreover, $K$ is a one-sided partial Heegaard surface for $M$, because there are no orientable incompressible surfaces in $M$ other than 2-spheres. (In [14] it is proved that $K$ is a one-sided Heegaard surface if $M$ is irreducible. Clearly the same argument works here.) By [12] there is a stable minimal incompressible surface $K^{\prime} \subset M$, with $K^{\prime}$ homeomorphic to $K$. $K^{\prime}$ is also a partial Heegaard surface in $M$. The preimage $\tilde{K}^{\prime}$ of $K^{\prime}$ in a double cover $\tilde{M}$ of $M$, as constructed in $\S 2$, is then an orientable minimal partial Heegaard surface for $\tilde{M}$. This completes the proof.

EXAMPLES. (1) The above hypotheses are satisfied by a finite connected sum $M=M_{1} \# \ldots \# M_{k}$, where each $M_{i}$ is either a copy of $S^{2} \times S^{1}$ or $\pi_{1}\left(M_{i}\right)$ is finite and at least one $\pi_{1}\left(M_{i}\right)$ is even, solvable and not generalised binary tetrahedral by cyclic.

(2) Let $M=L(4,1) \# S^{2} \times S^{1}$ with any Riemannian metric. Now $L(4,1)$ has an incompressible Klein bottle $K$ (see [14]) which can be minimally embedded in $M$, as in Theorem 5. $M$ has two double coverings, $\tilde{M}_{1}=R P^{3} \# S^{2} \times S^{1}$ and $\tilde{M}_{2}=$ $R P^{3} \# S^{2} \times S^{1} \# S^{2} \times S^{1}$, in which $K$ is covered by a minimal torus $\tilde{K}$ (cf. $\S 2$ ). In the former, the torus is nonseparating, while in the latter case, it is a separating surface. So we obtain all three types of partial Heegaard surfaces realized by minimal surfaces. 
(3) All the lens spaces $L(2 p, q)$ have embedded incompressible nonorientable surfaces $K$ (cf. [ 2 and 14] for properties of these surfaces). As in Theorem 5, $K$ can be chosen to be minimal and lifts to minimal Heegaard surfaces in both $L(p, q)$ and $S^{3}$. For example, with the standard spherical metric on $L(2 p, q)$ this produces many new embedded minimal surfaces in $S^{3}$, as compared with Lawson's constructions in [10]. See also [15] for a related procedure for finding minimal surfaces, based on an equivariant version of [12].

Note AdDed IN PROOF. Similar results have been obtained by S. Almeida, Ph. D. Thesis, State University of New York, Stony Brook (December 1982).

\section{REFERENCES}

1. J. S. Birman, On the equivalence of Heegaard splittings of closed orientable 3-manifolds, Ann. of Math. Studies (L. Neuwirth, ed.), no. 84, Princeton Univ. Press, Princeton, N.J., 1975, pp. 137-164.

2. G. Bredon and J. Wood, Nonorientable surfaces in orientable 3-manifolds, Invent. Math. 7 (1969), $83-110$

3. F. Bonahon and J-P. Otal, Scindements de Heegaard des espaces lenticulaires, preprint.

4. R. Engmann, Nicht-homöomorphe Heegaard-Zerlegungen vom Geschlecht 2 der zusammenhängen Summe zweier Linsenräume, Abh. Math. Sem. Univ. Hamburg 35 (1970), 33-38.

5. M. Gromov and H. B. Lawson, Jr., The classification of simply-connected manifolds of positive scalar curc'ature, Ann. of Math. (2) 111 (1980), 423-434.

6. M. Gromov and H. B. Lawson, Jr., Positive scalar curvature and the Dirac operator on complete Riemannian manifolds, preprint.

7. W. Haken, Some results on surfaces in 3-manifolds, Studies in Modern Topology (P. Hilton, ed.), Math. Assoc. Amer., Prentice-Hall, Englewood Cliffs, N. J., 1968, pp. 39-98.

8. R. S. Hamilton, Three manifolds with positive Ricci curvature, J. Differential Geom. 17 (1982), 255-306.

9. H. Kneser, Geschlossene Flächen in dreidimensionalen Mannigfaltigkeiten, Jahresber. Deutsch. Math.-Verein. 38 (1929), 248-260.

10. H. B. Lawson, Jr., Complete minimal surfaces in $S^{3}$, Ann. of Math. (2) 92 (1970), 335-374.

11. The unknottedness of minimal embeddings, Invent. Math. 11 (1970), 183-187.

12. W. Meeks, L. Simon and S-T. Yau, Embedded minimal surfaces, exotic spheres, and manifolds with positive Ricci curvature, Ann. of Math. (2) 116 (1982), 621-659.

13. J. Milnor, A unique factorization theorem for 3-manifolds, Amer. J. Math. 84 (1962), 1-7.

14. J. H. Rubinstein, One-sided Heegaard splittings of 3-manifolds, Pacific J. Math. 76 (1978), 185-200.

15. J. H. Rubinstein and L. Simon, Minimal surfaces invariant under groups of isometries in 3-manifolds (in preparation).

16. R. Schoen and S-T. Yau, On the structure of manifolds with positive scalar curvature, Manuscripta Math. 28 (1979), 159-183.

17. Existence of incompressible minimal surfaces and the topology of three-dimensional manifolds with non-negative scalar curvature, Ann. of Math. (2) 110 (1979), 127-142.

18. Complete three dimensional manifolds with positive Ricci curvature and scalar curvature, Ann. of Math. Studies (S-T. Yau, ed.), no. 102, Princeton Univ. Press, Princeton, N. J., 1982, pp. 209-228.

19. F. Waldhausen, Heegaard-Zerlegungen der 3-sphäre, Topology 7 (1968), 195-203.

Department of Mathematics, University of Melbourne, Parkville, Victoria 3052, Australia 